Transposing the term $X^{n}$ to the left-hand side and extracting the $n$th root of the two members of the resulting inequality, we find

$$
X \leqq \frac{\alpha}{2^{1 / n}-1} .
$$

The roots of largest absolute value $X$ are restricted by the double inequality

$$
\alpha \leqq X \leqq \frac{\alpha}{2^{1 / n}-1},
$$

where $\alpha$ denotes the largest of the quantities

$$
\left|a_{1}\right|,\left|a_{2}\right|^{1 / 2}, \cdots,\left|a_{n}\right|^{1 / n} \text {. }
$$

The inequality $X \geqq \alpha$ was noted by R. D. Carmichael and T. E. Mason,* who observed also that the lower limit is reached if the equation is

$$
(x+\alpha)^{n}=0 .
$$

It is also evident that the upper limit found above is reached if the equation is

$$
2 x^{n}-(x+\alpha)^{n}=0 .
$$

HARVARD UNIVERSITY, April 23, 1915.

\title{
CERTAIN NON-ENUMERABLE SETS OF INFINITE PERMUTATIONS.
}

\author{
BY PROFESSOR A. B. FRIZELL.
}

(Read before the American Mathematical Society April 10 and December 28,1914 .)

1. ThE simplest element of a permutation is the pairing of one of the objects permuted with a number indicating its place in the permutation. Such a pairing may be called a primitive element and denoted by $(i, n)$, where $n$ is the object and $i$ the number of the place assigned to it. In this paper the objects will all be numbers, finite or transfinite.

2. Permutations of finite sets are simply collocations of primitive elements. They are conveniently denoted by ex-

* Bulletin, vol. 21 (1914), pp. 14-22; in particular p. 20. 
pressions of the form $\left(i_{1}, n_{1}\right) \bigcirc\left(i_{2}, n_{2}\right) \bigcirc \cdots \circ\left(i_{k}, n_{k}\right)\left(i_{1}<i_{2}\right.$ $<\cdots<i_{k}$ ) and may be said to form polynomial elements. The number of the place will be called the index of a term (or monomial element) and the number placed there its digit. Index and digit may be transfinite and the terms may form an infinite series instead of a finite polynomial.

3 . If in an infinite well ordered set only a finite number of objects are permuted, the pairing of these objects with their new places may be called a finite permutation. It is easy to see that any set of finite permutations can be well ordered. If, e.g., in the series $1,2, \cdots$ we bring the numbers $2,3, \ldots$ successively to the first place, leaving the rest unchanged, and in each resulting series do likewise for the second place, then similarly for the third, and so on, the whole set is well ordered by the process of formation.

4. By an infinite permutation is to be understood here a pairing whereby infinitely many objects change their places. Thus, for example

$$
(1,2) \circ(2,1) \bigcirc(3,4) \bigcirc(4,3) \circ \cdots
$$

is an infinite permutation of the series $1,2, \ldots$ yielding the new series $2,1,4,3,6,5, \cdots$. Another example is obtained by interchanging the powers of 2 with the corresponding powers of 3 , giving the series $1,3,2,9,5,6,7,27,4,10,11, \cdots$.

5 . The polynomial elements that can be made with an $\omega$-series of digits and an $\omega$-series of indices form an enumerable set, since they are in one-to-one correspondence with the set of algebraic polynomials in which all coefficients are natural numbers. Let us order these polynomials according to the rules

$$
\begin{gathered}
(i, m)<(i, n) \quad \text { if } m<n ; \\
\left(i_{1}, m\right)<\left(i_{2}, n\right) \quad \text { if } i_{1}<i_{2} ;
\end{gathered}
$$

(3) $p<q$ if $p$ has fewer terms than $q$;

(4) $p<q$ if they have the same number of terms but the first term of $p$ that is not in $q$ is lower than the first term of $q$ which is not contained in $p$.

It is not difficult to show inductively that in this way the set is well ordered. Its type of order is the same as that which results from arranging the natural numbers according 
to their prime factors-first simple primes, then products of two primes, of three primes, and so on. This observation also proves at once that the set is countable.

6 . The polynomial elements $\Sigma(i, n)$ are now to be used in forming infinite permutations. In the series $1,2, \cdots$ let the monomial $(i, n)$ interchange an $\omega$-series of products of $n$ primes with a similar series of products of $i$ primes, and let the polynomial $\Sigma(i, n)$ effect simultaneously the exchanges indicated by its several terms. Thus the first primitive $(1,1)$ is an identical transformation, leaving the series unchanged; the second monomial $(1,2)$ is to interchange the simple primes with some $\omega$-series of products of two primes; let it be the first one, $2 p_{i}$, and we get the permutation $1,4,6,2,10,3$, $14,8,9,5,22,12,26,7,15,16,34,18,38,20, \cdots$ The transformation numbered $\omega$ is $(2,1)$; let it exchange the simple primes and the series $3 p_{i}$. The next transformation is $(2,2)$; let it interchange the series $2 p_{i}$ and $3 p_{i}$, yielding the series $1,2,3,9,5,15,7,8,4,21,11,12,13,33,6,16,17,18$, $19,20,10, \cdots$. We have, then, an enumerably infinite set of infinite permutations, since to every polynomial has been assigned a finite set of transpositions as just described. Whether these transpositions are all used does not interest us.

7. It is now easy to obtain non-denumerable sets by repeating the above process. To illustrate, let $(1,2)$ operate on the series of primes in the result of the transformation $(2,2)$; it produces the series $1,2,7,9,13,15,3,8,4,21,29$, $12,5,33,6,16,43,18,19,20,10,39, \cdots$ Let $p_{i}{ }^{\left({ }^{\circ}\right)}$ denote a polynomial and $P_{i}{ }^{(0)}$ the result of applying it as in $\S 6$, where $i$ is the ordinal symbol assigned to $p_{i}{ }^{(0)}$ by the rules of $\S 5$, and let the $\omega$-series used in $\$ 6$ be numbered likewise. Then $P^{(1)} \sim(i, n)$ shall be the permutation obtained by applying $p_{i}{ }^{(0)}$ as a transformation to the series whose ordinal symbol is $n$. The new symbols $(i, n)$ are to be combined into new polynomials $p^{(1)}$ as in $\S 2$ and ordered by the rules of $\S 5$. The new set of permutations $P^{(1)}$ cannot be put into one-to-one correspondence with set $\left[P^{(0)}\right]$. For on this assumption there still exists a $P^{(1)}$ which differs from $P_{1}{ }^{(1)}$ in the transformation $p^{(0)}$ performed on its first $\omega$-series, differs at the same time from $P_{2}{ }^{(1)}$ in the transformation performed on its second $\omega$-series, and from $P_{n}{ }^{(1)}$ in that performed on its $n$th $\omega$-series (where $n$ runs through the values $1,2, \cdots \omega, \cdots, \omega^{2}, \cdots$ ), and is therefore not contained in the assumed list, which is a contradiction. Hence $\left[P^{(1)}\right]$ is not enumerable. 
8. It is worth while to study the preceding types of order in a different way, following Cantor; they are all comprehended under the concept of a "Belegung." In forming the primitive elements of the $p^{(0)}$, two places are covered with an $\omega$-series of numbers; this covering is denoted by $\omega^{2}$. For the binomials, four places $i_{1}, n_{1}, i_{2}, n_{2}$ are covered with an $\omega$-series, indicated by $\omega^{4}$. The polynomials of $N$ terms have $2 N$ places to cover, giving the ordinal symbol $\omega^{2 N}$, whence the whole set $\left[p^{(0)}\right]$ is of ordinal type $\omega^{\omega}$. And this is the same as the type of the set of $\omega$-series of prime factors in the system of natural numbers, for the products of two primes exhibit a single $\omega$-series of $\omega$-series, $2 p_{i}, 3 p_{i}, 5 p_{i}, \cdots$; in the products of three primes $p_{\iota_{1}} \cdot p_{i_{2}} \cdot p_{i}$ the successive $\omega$-series are obtained by covering two places $i_{1}$ and $i_{2}$ with an $\omega$-series, with four factors three places are covered, and so on. The same type again is exhibited in each $P^{(0)}$ (cf. $\S 5$ ) and therefore in forming the $P^{(1)}$ we have likewise a covering. In the $P^{(1)}$ each $\omega$-series may be re-arranged as an $\omega^{\omega}$-series and this process admits an $\omega$-series of repetitions, but no more; beyond this point it yields nothing new.

There is a bit of formalism in the preceding which perhaps calls for closer scrutiny. The set of products of $N$ primes is in the strict sense a covering of $N$ factors with an $\omega$-series of values. Think of a row of $N$ boxes or spaces into each of which a compositor throws a prime. The totality of different ways in which he might set up this line is precisely what is meant by the symbol $\omega^{N}$. The symbol $\omega^{\omega}$, then, would mean, by analogy, that to every one of an $\omega$-series of spaces is assigned arbitrarily a prime number. But if an $\omega$-series of places is covered in this way with the series of primes we certain y do not get the set of natural numbers. And the covering of an $\omega$-series with an $\omega$-series is not a "limit" of the coverings $\omega^{N}$, because the set $\left[p^{(0)}\right]$ lies between them.. The use of $\omega^{\omega}$ for this set is merely a convenient symbolism not capable of supporting any logical deductions.

9 . The process by which the permutations $P^{(1)}$ were obtained from the $P^{(0)}$, however, being carried out by finite arithmetical steps, is not liable to inconsistency and, moreover, can be repeated. Thus we get a new set $\left[P^{(2)}\right]$, the digit $n$ running through the series of all ordinal values lower than the ordinal type of the set of series in $P^{(1)}$. Suppose that to every $P^{(2)}$ has been assigned a $P^{(1)}$. There exists a $P^{(2)}$ from which 
every $P_{\nu}{ }^{(2)}$ differs in its $\nu$ th transformation $\left(i_{\nu}, n_{\nu}\right)$ for values of $\nu$ forming a series of type $>\omega$. Hence the terms in the corresponding polynomial $p^{(2)}$ are a set whose type is $>\omega$. But there are no such polynomials. Therefore the $P^{(2)}$ cannot be put into one-to-one correspondence with the $P^{(1)}$. The same reasoning holds for the $P^{(1)}$, so we have two proofs that this set is non-enumerable.

10. The process of $\$ 7$ applied to the permutations $P^{(2)}$ yields a new set $\left[P^{(3)}\right]$ and the reasoning of $\$ 9$ shows that this set is not equivalent to $\left[P^{(2)}\right]$. Therefore by strict induction from $N$ to $N+1$ we infer the existence of an $\omega$-series of sets of infinite permutations no one of which can be put into one-to-one correspondence with its predecessor. Ordinally $\left[P^{(N+1)}\right]>\left[P^{(N)}\right]$ for $N=0,1,2, \cdots$. In Cantor's terminology, the set of infinite permutations of a simple infinity of objects presents an ordinal type higher than any finite aleph.

McPherson, Kansas.

\section{GEORGE WILLIAM HILL, 1838-1914.*}

George William Hill was the son of John William Hill and Catherine Smith, and was born in New York City on March 3, 1838. Both his father and grandfather were artists and he himself was of English and Huguenot descent. His early education like that of most of the men of his time in America gave him few advantages. In 1846, when his father moved from New York to the farm at West Nyack, the country was too busy with material development to produce many teachers who could give any but the most elementary instruction, and the country school which he attended must have been inferior in this respect to those of the larger cities. Even at Rutgers College in New Jersey, to which Hill was sent owing to the exhibition of unusual capacity and from which he took his degree in 1859 , the course probably went but little beyond that now found in secondary schools. There, however, he came under the influence of a man whose ideas on education were unusual. Dr. Strong, according to Hill's evidence, believed only in the classic treatises; but little published after

* Reprinted, by permission, from the Proceedings of the Royal Society May 3, 1915. 